\title{
Über Salze des fünfwertigen Mangans
}

\author{
Von Hermann Lux
}

Aus dem Anorganisch-chemischen Laboratorium der Technischen Hochschule München

(Z. Naturforschg. 1, 281-283 [1946]; eingegangen am 11. Februar 1946)

Beim Eintragen von $\mathrm{MnO}_{2}$ und $\mathrm{Na}_{2} \mathrm{O}$ in eine $\mathrm{NaNO}_{2}-\mathrm{Schmelze}$ entsteht eine intensive Blaufärbung, die von einer Verbindung des 5-wertigen Mangans herrührt. Aus konź.Natronlauge kristallisiert das Hydrat $\mathrm{Na}_{3} \mathrm{MnO}_{4}+10 \mathrm{H}_{2} \mathrm{O}$ in Form hellblauer Prismen, die sich in konz. Natronlauge schwer, in konz. Kalilauge leicht lösen. Die Bildung von Mischkristallen mit Natriumphosphat, -arsenat und -vanadat wird beobachtet.

Beim Verdünnen oder Erwärmen der Lösung erfolgt Disproportionierung in Manganat(VI) und $\mathrm{MnO}_{2}$; in stärker alkalischer Lösung besteht das Gleichgewicht: $\mathrm{Na}_{2} \mathrm{MnO}_{4}$ $+\mathrm{MnO}_{2}+4 \mathrm{NaOH} \rightleftharpoons 2 \mathrm{Na}_{3} \mathrm{MnO}_{4}+2 \mathrm{H}_{2} \mathrm{O}$.

Die Darstellung von Manganat (V)-hydrat gelingt in einfacher Weise durch Reduktion von Manganat(VII) oder Manganat(VI) mit $\mathrm{Na}_{2} \mathrm{SO}_{3}$ in stark alkalischer Lösung; auch zahlreiche andere Reduktionsmittel führen zur 5-wertigen Stufe des Mangans, sofern man nur in stark alkalischer Lösung und in der Kälte arbeitet.

$\mathrm{W}_{\mathrm{m}}^{\mathrm{e}}$ enn man geschmolzenes $\mathrm{NaNO}_{2}$ bei etwa $500^{\circ}$ mit $\mathrm{MnO}_{2}$ und etwas $\mathrm{Na}_{2} \mathrm{O}$ oder $\mathrm{Na}_{2} \mathrm{O}_{2}$ versetzt, zeigt sich in kurzer Zeit eine kräftige, rein blaue Färbung der Schmelze, die so lange bestehen bleibt, als noch überwiegend unzersetztes $\mathrm{NaNO}_{2}$ vorhanden ist. In dem Maße, in dem sich der Übergang in $\mathrm{NaNO}_{3}$ vollzieht, wird die Farbe grünstichiger und entspricht schließlich der bekannten tiefgrünen Manganatfärbung, wie sie bei Verwendung von $\mathrm{NaNO}_{3}$ oder $\mathrm{KNO}_{3}$ sogleich entsteht.

Diese Beobachtung machte ich schon bei den allerersten Vorversuchen, als ich mir die Frage vorlegte, welche Beziehungen zwischen der Alkalität und der Manganatfärbung einer Schmelze bei Gegenwart von $\mathrm{MnO}_{2}$ als Bodenkörper bestehen. Über die Ergebnisse dieser Untersuchungen wird zusammen mit R. Frey an anderer Stelle berichtet werden.

Da die Blaufärbung der $\mathrm{NaNO}_{2}$-Schmelzen ungefähr die gleiche Intensität aufwies wie die Grünfärbung der $\mathrm{NaNO}_{3}$-Schmelzen, schien der Farbunterschied mit der Anwesenheit von NO zusammenzuhängen. Als sich jedoch verschiedentlich nach dem Abkühlen blauer Schmelzen eine intensiv blaue Kruste an der Wand des Tiegels vorfand, entschloß ich mich zu systematischen Versuchen, die bald zeigten, daß man Schmelzkuchen erhalten konnte, die namentlich in einer mittleren Schicht reichlich dunkelblaue Körner neben farblosem $\mathrm{NaNO}_{2}, \mathrm{NaNO}_{3}$ und $\mathrm{Na}_{2} \mathrm{O}$ enthielten. Man schmolz hierzu $\mathrm{NaNO}_{2}$ bei $500-550^{\circ}$ im Silbertiegel ein, trug pulverisiertes $\mathrm{KMnO}_{4}$, dann $\mathrm{Na}_{2} \mathrm{O}_{2}$ unter Umrühren ein und ließ nach etwa $10 \mathrm{Min}$. im Ofen abkühlen.
Die Untersuchung der blauen Substanz ergab nun folgendes ${ }^{1}$ :

Beim Zusammenbringen mit Wasser entstand sofort tiefgrünes Manganat, gleichzeitig schied sich reichlich $\mathrm{MnO}_{2}$ ab. Verdünnte Natronlauge oder konzentrierte Ammoniaklösung wirkten ebenso. Über Calciumchlorid blieb jedoch die feste Substanz tagelang unverändert, selbst wenn sie schon etwas Wasser angezogen hatte. In Übereinstimmung damit trat mit konzentrierter Natronlauge $(1+1)$ keine Veränderung ein; die blaue Substanz blieb zum größten Teil ungelöst, die schwach blau gefärbte Lauge hielt sich bei Ausschluß von $\mathrm{CO}_{2}$ und $\mathrm{H}_{2} \mathrm{O}$ beliebig lange. Sehr viel größer war die Löslichkeit in konz. Kalilauge $(1+1)$; beim Verdünnen der kräftig blauen, wie konz. $\mathrm{CuSO}_{4}$ Lösung aussehenden Lösung in Kalilauge entstand $\mathrm{MnO}_{2}$ und grünes Manganat. Auch beim Erwärmen vollzog sich die gleiche Reaktion; hier entstand zunächst eine leuchtend grüne, klare Lösung, die sich rasch durch Ausscheidung von $\mathrm{MnO}_{2}$ trübte.

Um festzustellen, in welchem Verhältnis $\mathrm{MnO}_{4}^{-2}$ und $\mathrm{MnO}_{2}$ entstanden, wurde die blaue Substanz in der Kälte zunächst mit Natronlauge $(1+1)$ verrieben, durch Filtration von dem in Lösung gegangenen grünen Manganat getrennt und auf dem Filter mit Kalilauge $(1+1)$ gelöst, wobei etwas $\mathrm{MnO}_{2}$ zurückblieb. Dann verdünnte man auf das Vierfache und filtrierte das ausgeschiedene $\mathrm{MnO}_{2}$ nach 10-30 Min. ab. Die colorimetrische Bestimmung

\footnotetext{
1 Sämtliche Angaben beziehen sich auf eine Arbeitstemperatur von $2-5^{0}$.
} 
des Mangans lieferte Werte, die dem Molverhältnis $1: 1$ sehr nahe kamen, z. B. $\mathrm{MnO}_{4}^{-2}: \mathrm{MnO}_{2}=$ $0,94: 1$ und $0,96: 1$. Genauere Utbereinstimmung konnte nicht erwartet werden, da die Lösung auch $\mathrm{NaNO}_{2}$ enthielt, das bei der angewandten Verdünnung der Lauge schon langsam mit $\mathrm{MnO}_{4}^{-2}$ reagierte. Ein weiterer Grund, warum sich nicht genau das Verhältnis $1: 1$ ergeben konnte, wird später zu nennen sein.

Die beschriebenen Versuche zeigten klar, daß es sich bei der blauen Substanz nicht etwa um eine NO-haltige Verbindung handelte, sondern daß vielmehr eine Verbindung des fünfwertigen Mangans vorliegen mußte, die beim Verdünnen der stark alkalischen Lösung unter Disproportionierung in Manganat(VI) und $\mathrm{MnO}_{2}$ zerfiel.

Ließ man die blaue Substanz mit Natronlauge $(1+1)$ über Nacht im Exsiecator stehen, so trat meist Kristallisation ein. Bei Anwendung von Natronlauge $(1+2,5)$ entstanden meist schon nach wenigen Sekunden gut ausgebildete hellblaue rechteckige Stäbchen, die ersichtlich weniger löslich waren als das wasserfreie Ausgangsprodukt. Wie noch gezeigt werden wird, handelt es sich dabei um ein wasserreiches Hydrat. Ließ man das auskristallisierte Hydrat mit der Lauge im Exsiccator über Ätznatron stehen, so bildeten sich offenbar wasserärmere, tiefer blaue Kristalle von eigentümlicher, kometenähnlicher Form. Kristallisierte Kaliumsalze wurden bisher nicht beobachtet.

Wenn es wirklich um fünfwertiges Mangan handelte, war zu vermuten, daß das krist. Hydrat in seiner Zusammensetzung den entsprechenden Hydraten des fünfwertigen Phosphors, Arsens oder Vanadiums ähnlich und vielleicht mit diesen isomorph war. Kristallisationsversuche sprechen dafür, daß diese Annahme zutrifft. Natriumphosphat, -arsenat und -vanadat kristallisieren aus Natronlauge gleicher Konzentration ebenfalls in Form rechteckiger Stäbchen. Läßt man Manganat(V) und Arsenat, Phosphat oder Vanadat nebeneinander kristallisieren, so beobachtet man unter dem Mikroskop eine Zone, in welcher der Grad der Anfärbung mit blauem Manganat(V) in stetiger Weise zunimmt.

Da Manganat (V) etwas weniger intensiv farbig ist als das tiefgrüne Manganat(VI), ist es neben diesem nur schwer zu erkennen. Dies dürfte auch der Grund sein, warum es sich bis heute der Beobachtung entzogen hat. Die eingehende Durchsicht der Literatur zeigte jedoch, daß V. A uger ${ }^{2}$ schon 1910 ein kristallines Produkt von bläulich grünem Aussehen beschrieben hat, das er durch Erhitzen von Manganat(VII) mit Ätznatron erhielt. Die Analyse des unreinen Salzes deutete auf die Formel $3 \mathrm{Na}_{2} \mathrm{O} . \mathrm{MnO}_{2} . \mathrm{MnO}_{3} \cdot \mathrm{H}_{2} \mathrm{O}$. Er betrachtete es wegen seines leichten Zerfalls als Doppelsalz mit 4- und 6-wertigem Mangan und bezeichnete es daher als Manganimanganat. Diese Auffassung wurde gestützt durch eine Arbeit von O. Sa ck u r ${ }^{3}$, nach welcher in geschmolzenen Alkalicarbonaten oder -hydroxyden weitgehend unabhängig von der Temperatur und den Mengenverhältnissen Verbindungen der Zusammensetzung $8 \mathrm{~K}_{2} \mathrm{O}$ - $2 \mathrm{MnO}_{2} \cdot 3 \mathrm{MnO}_{3}$ und $4 \mathrm{Na}_{2} \mathrm{O} \cdot \mathrm{MnO}_{2} \cdot \mathrm{MnO}_{3}$ entstehen. G. J u s t und Y. K a u k ${ }^{4}$ schlossen aus ihren Versuchen über die Reduktion von $\mathrm{KMnO}_{4}$ mit $\mathrm{H}_{2}$ oder $\mathrm{CO}$, daß 5-wertiges Mangan als instabile Zwischenstufe auftreten müsse. Zum gleichen Schluß kam J. H ollu ta ${ }^{5}$. Er nahm zur Erklärung der Kinetik der Reaktion zwischen Permanganat und Formiat ein Anion mit 5-wertigem Mangan als Zwischenstufe an und konnte später sogar feststellen, daß das Maximum der bei dieser Reaktion in stark alkalischer Lösung vorübergehend beobachteten Blaufärbung mit dem Erreichen des 5-wertigen Zustandes beim Mangan zusammenfiel. Gegen die Auffassung, daß die Blaufärbung von einem Manganimanganat-Komplex herrühre, führt $\mathrm{H}$ olluta an, daß es ihm nicht gelungen sei, aus hydratischem Braunstein und Manganat (VI) blaugefärbte Lösungen zu erhalten.

Die geringe Löslichkeit und das gute Kristallisationsvermögen des beschriebenen Manganat(V)hydrats ermöglichen es, Manganat(V) neben dem äußerst leicht löslichen Manganat(VI) zu erkennen. Es gelang so zu beweisen, daß die fünfwertige Stufe des Mangans keineswegs den Charakter einer instabilen Zwischenstufe hat, sondern im Gleichgewicht mit Manganat(VI) und $\mathrm{MnO}_{2}$ in stark alkalischem Medium völlig stabil ist. Bringt man. nämlich reaktionsfähiges $\mathrm{MnO}_{2}$ mit Manganat (VI) bei genügender $\mathrm{NaOH}-$ Konzentration zusammen, so bilden sich in kurzer Zeit die charakteristischen blauen Stäbchen des schwer löslichen Manganat(V)-hydrats. Man löst dazu $\mathrm{MnSO}_{4}$ in ganz wenig Wasser, fällt mit Natronlauge $(1+2,5)$ und oxydiert mit Manganat(VI)-Lösung gleicher Laugenkonzentration, bis eine schwache Grünfärbung bestehen bleibt. Bringt man dieses frisch hergestellte $\mathrm{MnO}_{2}$ auf dem Objektträger mit überschüssiger Manganat(VI)-Lauge zusammen, so treten meist schon nach wenigen Minuten die Manganat(V)-Stächen auf. Man kann dabei deutlich beobachten, wie der Braunstein besonders stark in der Umgebung der wachsenden Kristalle aufgezehrt wird. Mit käuflichem $\mathrm{MnO}_{2}$ gelingt die Reaktion nicht.

2 C. r. hebd. Séances Acad. Sci. 150, 470 [1910].

3 Ber. dtsch. chem. Ges. 44, 777 [1911].

4 Z. physik. Chem. 76, 638 [1911] ; 82, 71 [1913].

5 Z. physik. Chem. 106, 324 [1923]. 
Zwischen Manganat(VI) und $\mathrm{MnO}_{2}$ einerseits und Manganat( $V$ ) andererseits besteht somit ein Gleichgewicht

$$
\stackrel{+6}{\mathrm{Na}_{2} \mathrm{MnO}_{4}}+\stackrel{+4}{\mathrm{MnO}_{2}}+\underset{4 \mathrm{NaOH}}{\rightleftharpoons} \underset{2 \mathrm{Na}_{3} \mathrm{MnO}_{4}}{+5}+2 \mathrm{H}_{2} \mathrm{O}
$$

das dem Massenwirkungsgesetz entsprechend bei hoher NaOH-Konzentration so weit nach rechts verschoben ist, daß die Löslichkeit des Manganat(V)-hydrats überschritten wird. Die genauere Festlegung der Gleichgewichte und der zugehörigen Redoxpotentiale in Abhängigkeit von $\mathrm{NaOH}-$ Konzentration und Temperatur bleibt einer späteren Mitteilung vorbehalten.

Die Darstellung von Manganat(V)-hydrat gelingt sehr einfach: Mit einer Lösung von $2 \mathrm{~g}$ feinst pulverisiertem $\mathrm{KMnO}_{4}$ in $50 \mathrm{ccm}$ Natronlauge $(1+2,5)$ werden $3,5 \mathrm{~g}$ fein zerriebenes $\mathrm{Na}_{2} \mathrm{SO}_{3}$ $+7 \mathrm{H}_{2} \mathrm{O}$ bei $0^{\circ}$ etwa $10 \mathrm{Min}$. verrührt, bis das Ganze einheitlich heliblau geworden ist. Dann saugt man scharf ab und wäscht mit Natronlauge $(1+2,5)$ nach.

Lösungen von Manganat(VII) oder Manganat (VI) in Natronlauge $(1+2,5)$ werden in der Kälte durch $\mathrm{Na}_{2} \mathrm{SO}_{3}, \mathrm{NaOOCH}$ oder $\mathrm{Na}_{2} \mathrm{~S}_{2} \mathrm{O}_{3}$ quantitativ bis zur 5-wertigen Stufe reduziert. Bei mehreren anderen Reduktionsmitteln tritt blaues Manganat(V) nur vorübergehend als $Z$ wischenstufe auf. Besonders hübsch sieht die Reaktion von $\mathrm{KMnO}_{4}$ mit $\mathrm{NaBO}_{2} \cdot \mathrm{H}_{2} \mathrm{O}_{2}$ aus; hier zeigen sich innerhalb von 1-2 Min. alle vier Wertigkeitsstufen des Man- gans (rotviolett - tiefgrün - himmelblau braungelb). Eingehende Versuche haben gezeigt, daß die fünfwertige Stufe des Mangans etwa ebenso häufig auftritt wie andere Wertigkeitsstufen, sofern man nur in stark alkalischer Lösung und in der Kälte arbeitet.

In dem dargestellten Manganat(V)-hydrat waren $\mathrm{SO}_{4}-2$ und $\mathrm{K}+$ nicht enthalten. Die jodometrische $\mathrm{Be}-$ stimmung des Oxydationswertes ergab 3,01 val/g-atom Mn entspr. der Wertigkeitsstufe 5,01. Zur Analyse wurde Mangan durch Ammoniak und Brom abgetrennt; Mangan wurde als $\mathrm{MnSO}_{4}$, Natrium als $\mathrm{Na}_{2} \mathrm{SO}_{4}$ gewogen. Bei zwei verschiedenen Präparaten ergaben sich folgende Werte:

$$
\begin{array}{rccc} 
& \mathrm{Na}_{2} \mathrm{O} & \mathrm{Mn}_{2} \mathrm{O}_{5} & \mathrm{H}_{2} \mathrm{O} \text { (Differenz) } \\
\text { I. } & 23,50 & 16,14 & 60,36 \text { Gew.- } \% \\
\text { II. } & 23,78 & 16,41 & 59,81 \text { Gew. } \%
\end{array}
$$

Beiden Präparaten haftete Natronlauge an, deren Gehalt zu 20,88 Gew.-\% $\mathrm{Na}_{2} \mathrm{O}$ bestimmt wurde. Die gefundenen Analysenwerte müssen, in ein Koordinatensystem eingetragen, auf einer Geraden liegen, welche die Punkte der Zusammensetzungen von Natronlauge und gesuchtem Hydrat miteinander verbindet. Beide Analysenwerte liégen nun recht genau auf der Verbindungslinie zum Punkt $\mathrm{Na}_{3} \mathrm{MnO}_{4}+10 \mathrm{H}_{2} \mathrm{O}$; theoretische Werte sind z. B. $23,7 \% \mathrm{Na}_{2} \mathrm{O}, 16,2 \% \mathrm{Mn}_{2} \mathrm{O}_{5}, 60,1 \% \mathrm{H}_{2} \mathrm{O}$. Für das $12-\mathrm{Hy}-$ drat, dessen Vorliegen zunächst vermutet worden war, sollte bei gleichem Mangangehalt gefunden werden: $22,4 \% \mathrm{Na}_{2} \mathrm{O}, 16,2 \% \mathrm{Mn}_{2} \mathrm{O}_{5}, 61,4 \% \mathrm{H}_{2} \mathrm{O}$. Wie man erwarten darf, scheint somit das 5-wertige Mangan dem 5-wertigen Vanadium besonders ähnlich zu sein, das ebenfalls Hydrate mit $10 \mathrm{H}_{2} \mathrm{O}$ bildet. Uber die Mischkristallbildung zwischen Natriummanganat(V) einerseits und tertiärem Natriumarsenat, -phosphat und -vanadat andererseits sowie über die Hydratstufen sind noch eingehendere Untersuchungen erforderlich.

\title{
Zuf Biochemie der Schilddrüsenfunktion, III. Mitteilung: Die entgiftende Wirkung des Vitamins C gegenüber kropferzeugenden Substanzen
}

\author{
Von Friedrich HÜter \\ Aus dem Institut für Chemotherapie „Georg-Speyer-Haus“, Frankfurt a. M. \\ (Z. Naturforschg. 1, 283-287 [1946]; eingegangen am 8. April 1946)

\begin{abstract}
Der Schilddrüsenreizstoff des Weißkohles erzeugt im lebenden Organismus einen relativen C-Vitaminmangel. Die Ascorbinsäure wirkt hinsichtlich strumigener Substanzen kropfhemmend. Das rasche Entstehen einer mehrfach vergrößerten, basedowähnlichen Schilddrüse durch eine pflanzliche Noxe plus Allylthioharnstoff wird beschrieben.
\end{abstract}

$\mathrm{M}$ a rine und Mitarbeiter ${ }^{2}$ berichten über einen kropfhemmenden Pflanzenwirkstoff und nehmen an, das Vitamin $\mathrm{C}$ übe vielleicht diese antagonistische Wirkung aus; sie bringen aber dafür

${ }^{1}$ I. Mitteilg.: Th. Wagner-Jauregg u. E. Schrei be r, Biochem. Z. 317, 21 [1944]; II. Mitteilg.: Th. Wa g ner-Jauregg u. J. Koch, unveröffentl. keine experimentellen Beweise. Nach ihren Befunden führt gedämpfter und abgepreßter Kohl zu einer stärkeren Schilddrüsenwucherung als frischer. Wir prüften mit handelsüblichem Weißkraut

$2 \mathrm{M}$ a r in e, B a u m a n , Webster u. Cipra, J. exp. Medicine 57, 121 [1933]; ferner J. biol. Chemistry 89, $213[1930]$. 